\title{
Diagnostic des causes de complication du diabète et des méthodes de prévention à Bobo-Dioulasso, au Burkina Faso
}

\author{
Kossi-Kuma Agbalebon KOEVI ${ }^{1}$, Vinsoun MILLOGO ${ }^{1 *}$, Macaire OUEDRAOGO ${ }^{2}$ et \\ Georges Anicet OUEDRAOGO ${ }^{1}$
}

\begin{abstract}
${ }^{1}$ Département de Production Animale, Laboratoire de Recherche et d'Enseignement de Recherche en Santé et Biotechnologie Animales (LA.R.E.S.B.A), Institut de Développement Rural, Université Polytechnique de BoboDioulasso, 01 BP 1091, Bobo-Dioulasso 01, Burkina Faso.

${ }^{2}$ Département de Médecine, Service de Médicine Interne, Centre Hospitalier Universitaire Sourô Sanou (CHUSS) de Bobo-Dioulasso, 01 BP 676, Bobo-Dioulasso 01, Burkina Faso.

*Auteur correspondant ; E-mail : paravins@yahoo.fr ; Tel : +226 70228995 ; +226 75229209
\end{abstract}

\section{RESUME}

Au Burkina Faso, on assiste à une progression du diabète due, en grande partie, à l'ignorance, aux nouvelles et mauvaises habitudes alimentaires. L'objectif de notre étude était de diagnostiquer les causes de complications de cette maladie afin de les prévenir. Notre étude a été conduite à Bobo-Dioulasso de mars à septembre 2012. Les enquêtes et des mesures biologiques ont été réalisées sur 574 patients diabétiques. Les statistiques descriptives et les tests de corrélation ont été faits à l'aide du logiciel SPSS 18. Des patients rencontrés, $89 \%$ avaient plus de 40 ans parmi lesquels $66 \%$ de femmes et $47 \%$ de ménagères. L'âge, le sexe, la profession, le revenu et l'antécédent familial étaient les facteurs de risques d'apparition du diabète. Des corrélations positives ont été observées entre l'hypertension artérielle et l'âge $(\mathrm{r}=0,340 ; \mathrm{P}=0,000)$ d'une part et la profession $(\mathrm{r}=0,142 ; \mathrm{P}=0,001)$ d'autre part ; entre l'artériopathie et la dyslipidémie $(\mathrm{r}=0,332 ; \mathrm{P}=$ $0,000)$ et entre l'artériopathie et l'hyper-uricémie $(r=0,147 ; \mathrm{P}=0,000)$. Les personnes âgées de plus de 40 ans doivent se soumettre à une activité physique régulière et à une alimentation équilibrée qu'elles soient diabétiques ou pas.

(C) 2014 International Formulae Group. All rights reserved.

Mots clés: Burkina Faso, diabète, causes de complications, prévention.

\section{INTRODUCTION}

Le diabète sucré est un groupe d'affections métaboliques, caractérisées par une hyperglycémie chronique résultant d'un défaut de sécrétion, d'action d'insuline ou des deux (Grimaldi et al., 1998). Dans le monde, une personne meurt du diabète toutes les dix secondes, au cours de ces mêmes 10 secondes, deux cas sont diagnostiqués (IDF, 2009). On estime à environ $4 \%$ de décès imputés à cette maladie (IDF, 2009). Selon les projections de l'OMS, le nombre de décès dû au diabète va doubler entre 2005 et 2030 (IDF, 2009). L'Afrique est le continent le moins touché avec environ 12,10 millions de diabétiques enregistrés en 2010. Malheureusement, pour son taux de croissance de la morbidité du diabète sucré de $98 \%$ (le plus élevé du monde) 
ces 20 prochaines années, l'Afrique comptera à l'horizon 2030 environ 23,90 millions de diabétiques (IDF, 2009). La mortalité attribuable au diabète en Afrique subsaharienne est estimée, en 2010, à 6\% de la mortalité totale (IDF, 2009). En 2030, au Burkina Faso, la prévalence se situerait entre 4 et 5\% (IDF, 2009). Une étude réalisée au Centre Hospitalier Universitaire Yalgado Ouedraogo par Sano et al. (1999) a montré que la prévention des lésions de pied pourrait être efficace à condition qu'elle soit intégrée dans une stratégie globale de prise en charge des diabétiques. Une autre étude menée dans ce même centre par Ouedraogo et al. (2000) a montré que l'hyperglycémie chronique, les écarts de régime, les erreurs thérapeutiques, les infections ont été responsables de ces complications aigües dont le taux de létalité a été de 8,2\%. Toujours au Burkina Faso, beaucoup de travaux de recherche ont été effectués pour évoquer les complications du diabète notamment à Ouagadougou et à BoboDioulasso, alors la prise en charge a été renforcée par la formation de mise à niveau des médecins traitants et la disponibilité des médicaments génériques antidiabétiques dans toutes les régions du pays (Drabo et al., 2011). Malgré ces interventions, le diabète fait toujours des victimes au sein de la population. Une étude menée par Horde (2011), montre que près de $90 \%$ des diabétiques vivent pendant des années avec cette maladie sans le savoir car le diabète ne provoque en général pas de manifestations pendant une très longue période. Dans bien des cas, les symptômes sont tellement mineurs qu'ils passent inaperçus pendant plusieurs années. On estime qu'il faut en moyenne sept ans pour qu'un diagnostic soit posé par un médecin. Ceci traduit la nécessité d'avoir une attention toute particulière quant au diagnostic précoce et à la prise en charge adéquate du diabète. Alors, la connaissance de la situation réelle de la survenue du diabète à Bobo-Dioulasso permettra-t-elle de le prévenir? Ainsi, notre hypothèse est que la connaissance de la survenue du diabète nous permettra de mener une intervention précoce pour freiner l'évolution de celui-ci. L'objectif général de ce travail était de diagnostiquer les causes de complication et de prévenir le diabète. Pour ce faire, nous avons retenu comme objectifs spécifiques : d'identifier les différents facteurs intrinsèques et extrinsèques qui concourent à l'apparition du diabète, de décrire les circonstances de découvertes du diabète, de déterminer l'efficience des méthodes de prévention adaptées au contexte.

\section{MATERIEL ET METHODES}

\section{Sites de l'étude}

La présente étude a été conduite dans la ville de Bobo-Dioulasso, chef-lieu de la Région des Hauts-Bassins, deuxième grand centre urbain du Burkina Faso, de mars à septembre 2012. La population était de 813 000 habitants selon les données de INSD / EPB (Institut National de la Statistique Démographique /Etude de la Population de Bobo-Dioulasso, 2012) et la prévalence du diabète a été estimée à $4 \%$ (IDF, 2009). Les enquêtes ont été réalisées dans le département de médecine du Centre Hospitalier Universitaire Sourô Sanou (CHUSS), dans le dispensaire du Centre d'Accueil, d'écoute et d'éveil des personnes âgées (Burkina Secours) et au Centre Médical Social (CMS) Don Bosco. La fréquentation des diabétiques dans ces centres en 2010 a été de $13 \%$, 5\% et de $2 \%$ respectivement.

\section{Matériel biologique}

Le patient diabétique a été considéré comme le sujet biologique. Le patient diabétique est une personne chez qui on a reconnu un groupe d'affections hétérogènes avec les éléments communs d'une 
hyperglycémie et d'une intolérance au glucose, en raison d'une carence en insuline, de troubles de l'efficacité de l'action de l'insuline ou des deux.

\section{Echantillonnage}

Cette étude a été axée sur la description du passé du patient et sur l'exploitation de leur dossier médical. En tenant compte de la prévalence du diabète au Burkina Faso et en faisant une extrapolation sur la ville de BoboDioulasso en fonction de sa population, alors, nous avons une valeur approximative de 32545 diabétiques déclarés ou non déclarés. En tenant compte de la fréquentation des diabétiques au CHUSS comme centre de référence estimée à $13 \%$ en 2010 , alors nous avons 4231 diabétiques. De cette population a été extraite la population cible qui a constitué notre échantillon d'étude. Ainsi, la taille de l'échantillon a été estimée à 215 patients diabétiques pour une marge d'erreur de $5 \%$. L'échantillon définitif de cette étude était de 574 personnes diabétiques dont l'âge était compris entre 18 et 85 ans avec une moyenne de $55 \pm 12$ ans, soit le double de notre estimation. Les enfants et les patients diabétiques hospitalisés n'ont pas été pris en compte dans l'échantillonnage. La participation des patients diabétiques se fait sur la base du consentement écrit. En effet, une lettre de consentement fut envoyée aux personnes ciblées et c'est à la suite de leur réponse et accord qu'elles ont été définitivement retenues pour l'étude. Après l'élaboration des lettres de consentement, celles-ci étaient envoyées par courrier officiel aux différents centres concernés par l'étude. Il revenait aux centres sanitaires de faire la répartition des lettres individuelles de consentement de façon aléatoire. La remise des lettres de consentement se faisait pendant les consultations et un saut de deux patients était fait pour la remise. Le taux de retour des lettres favorables a été estimé à 78\%. En outre, vu que l'étude a été soumise à un comité d'éthique, les informations sur les personnes impliquées dans cette étude sont restées confidentielles.

\section{Elaboration des questionnaires}

L'étude a été focalisée sur les causes des complications et des méthodes de traitement du diabète. Les questions ont été axées sur l'âge, le sexe, la profession, le revenu mensuel, les antécédents familiaux, la durée de l'évolution du diabète, la circonstance de découverte du diabète, le tabagisme (la durée et le nombre de cigarettes fumées / jour), la consommation de l'alcool. Les données ont été mentionnées sur une fiche de collecte.

\section{Administration des questionnaires}

Les questionnaires ont été administrés par interview direct des patients pendant les consultations. Les patients ont été reçus individuellement en consultation médicale. Les questions ont été posées clairement aux patients pour leur faciliter la compréhension sans aucune contrainte. Le patient, sans être sous pression, avait le droit de répondre partiellement ou entièrement à toutes les questions qui lui avaient été adressées.

\section{Mesures biologiques}

Des mesures biologiques ont été faites et les constantes biologiques telles que le poids, la taille, la tension artérielle et la circonférence de l'abdomen chez chaque participant diabétique ont été effectués. La pression artérielle a pu être mesurée grâce au tensiomètre (Elégance CT-BP20, Allemagne, 2009); le pèse-personne (Seca France, 2009) a permis de mesurer le poids de chaque patient. Le mètre ruban nous a permis de mesurer la circonférence de l'abdomen des patients. Quant à la taille, elle a été considérée à partir de la carte d'identité nationale du patient. 


\section{Analyses statistiques}

Les réponses des questionnaires ont été codées et mentionnées sur une fiche qui a permis à la fin de l'enquête d'effectuer le dépouillement. La saisie des résultats du dépouillement a été effectuée sur le logiciel EXCEL $2007^{\circledR}$.

L'analyse des données a été effectuée à l'aide du logiciel SPSS 18 pour WINDOWS 7. Les statistiques descriptives ont permis d'apprécier les variables de tendances centrales et de dispersion. Une approximation a été faite sur les variables suivant la loi normale et ensuite une analyse de variance (ANOVA) a été effectuée. Les moyennes ont été comparées en utilisant le test de Student et les différences ont été considérées comme significatives au seuil de probabilité $\mathrm{p}<0,05$. Par ailleurs, le test de corrélation (corrélation de Pearson) a été utilisé pour montrer les liens probables entre les facteurs extrinsèques et intrinsèques qui concourent à l'apparition de la maladie. Quant au coefficient de corrélation de Pearson, il a été utilisé pour l'étude de la corrélation cause-effet.

\section{RESULTATS}

\section{Facteurs intrinsèques et extrinsèques d'apparition du diabète}

Les résultats indiquent que $89 \%$ des patients diabétiques rencontrés avaient plus de 40 ans (Tableau 1). On note également que les femmes représentaient $66 \%$ des diabétiques rencontrés. En plus, d'autres facteurs extrinsèques se dégageaient à l'issue des enquêtes. En effet, on observe que $47 \%$ des personnes rencontrées étaient ménagères.

En prenant l'âge, le sexe, la profession, le revenu et l'antécédent familial, on peut prévoir en tenant compte des coefficients de corrélation, les risques d'apparition du diabète chez une personne dans la population de Bobo-Dioulasso (Tableau 1 et Tableau 3).
Les facteurs intrinsèques (l'âge et le sexe) ont pu enregistrer bon nombre de corrélations. Ainsi une corrélation significative $(\mathrm{p}<0,01)$ a été notée entre l'âge et l'HTA $(0,340$ avec $\mathrm{p}=0,000)$, la rétinopathie $(0,169$ avec $\mathrm{p}=0,000)$, le maux d'oreille $(0,117$ avec $\mathrm{p}=0,008)$, perte de dents $(0,324$ avec $\mathrm{p}=0,000)$, l'IMC $(0,120$ avec $\mathrm{p}=0,004)$ et beaucoup de corrélation significative à $\mathrm{p}<0,05$ ont été également notées entre l'âge et la dyslipidémie $(0,102$ avec $\mathrm{p}=0,014), \mathrm{l}^{\prime} \mathrm{HbA}_{1} \mathrm{C}(0,097$ avec $\mathrm{p}=$ $0,020)$, créatinémie $(0,107$ avec $\mathrm{p}=0,010)$.

En plus, des corrélations significatives ( $\mathrm{p}<0,01)$ ont été observées entre le sexe et l'IMC (0,290 avec p = 0,000), l'obésité (0,219 avec $\mathrm{p}=0,000)$, le tour de taille $(0,184$ avec $\mathrm{p}$ $=0,000)$, le trouble sexuel $(0,292$ avec $\mathrm{p}=$ $0,000)$ et à $\mathrm{p}<0,05$ entre le sexe et la protéine de 24 heures $(0,093$ avec $\mathrm{p}=0,026)$.

Il est à noter que les facteurs extrinsèques (profession et revenu mensuel) ont peu de corrélation avec les paramètres cliniques et biologiques des patients. A cet effet, la profession a une corrélation avec l'HTA à $0,342(\mathrm{p}=0,001)$, avec la créatinémie à $0,096(\mathrm{p}=0,021)$ et avec la perte de dents à $0,098(\mathrm{p}=0,019$. Le revenu mensuel quant à lui a une corrélation significative avec la perte de dents à $0,093(\mathrm{p}=0,027)$, avec le trouble sexuel à $0,142(\mathrm{p}=0,001)$ avec l'angor / cardiopathie à $0,103(\mathrm{p}=0,013)$ (Tableau 3).

\section{Circonstances de découvertes du diabète}

Les résultats indiquent que la tension artérielle au niveau du bras droit était de $142,61 \pm 25,22$ en $\mathrm{mm} \mathrm{Hg}$ (systole) et de $82,77 \pm 13,9$ de $\mathrm{mm} \mathrm{Hg}$ (diastole) et le pouls a donnée $83,87 \pm 17,63$ battements/minute pour les 574 patients.

Quant au bras gauche, les résultats indiquent $139,01 \pm 23,95 \mathrm{~mm} \mathrm{Hg}$ (systole) et $80,98 \pm 12,76 \mathrm{~mm} \mathrm{Hg}$ (diastole) pendant qu'on notait $83,59 \pm 17,39$ de 
battements/minutes pour le pouls pour l'ensemble des 574 patients.

Les composantes lipidiques responsables de la dyslipidémie chez les patients ont donné $4,92 \pm 1,10$ en $\mathrm{mmol} / \mathrm{L}$ pour le cholestérol, à $2,35 \pm 0,38 \mathrm{mmol} / \mathrm{L}$ pour le HDL-cholestérol, à $2,08 \pm 0,87 \mathrm{mmol} / \mathrm{L}$ pour le LDL-cholestérol et à $2,45 \pm 0,74 \mathrm{mmol} / \mathrm{L}$ pour les triglycérides.

Les résultats ont donné pour l'acide urique une valeur de 352,23 $\pm 140,65 \mu \mathrm{mol} / \mathrm{L}$ et $9,47 \pm 2,41 \%$ pour l'hémoglobine glyquée $(\mathrm{HbA} 1 \mathrm{C})$ à $66,82 \pm 20,34 \mu \mathrm{mol} / \mathrm{L}$ pour la créatinémie. Quant à l'Indice de Masse Corporelle (IMC), la valeur moyenne obtenue pour l'ensemble des patients est de 26,90 \pm $5,80 \mathrm{~kg} / \mathrm{m}^{2} ; 94,29 \pm 13,02 \mathrm{~cm}$ pour le tour de taille chez les hommes et de 94,20 $\pm 12,65 \mathrm{~cm}$ chez les femmes. La perte de dent a été très marquée avec une moyenne de 4,54 $\pm 6,41$ au sein de la population étudiée (Tableau 2).

Par ailleurs, des complications ont été décelées chez $80,1 \%$ des patients. Parmi ces complications, $14,3 \%$ des patients étaient atteints de polyurie ; $10,1 \%$ par la polyurie et l'amaigrissement, 8,7\% par la polyurie et la polydipsie $5,1 \%$ par l'amaigrissement et la polydipsie.

Des résultats d'autres complications rencontrées montrent que $4,2 \%$ des patients avaient une hypertension artérielle; $3 \%$ une hypertension et polyurie; $2,3 \%$ des douleurs articulaires ; 2,3\% des douleurs des doigts. En plus, on observe aussi que $2,3 \%$ des patients avaient l'hypertension artérielle et des urines jaunes; 2,3\% avaient la polyurie, l'amaigrissement, la polydipsie et la polyphagie et $1,6 \%$ avaient la polydipsie; la polyurie, la polydipsie et $1,4 \%$ avaient la polyphagie; $1,2 \%$ avaient la Polyurie et des maux des yeux; $1 \%$ était atteint d'hypertension artérielle, de polyurie et de polydipsie. De plus, de rares cas $<1 \%$ de crampe des doigts, d'inflammation des pieds, de plaie plantaire, de crampes plantaires; de rares cas de moins de $1 \%$ de plaie des orteils, des vertiges, de plaie non cicatrisée, de lombalgie, de douleurs plantaires, d'angine de poitrine, de tremblement et de rares cas de moins de $1 \%$ de transpiration de grosse sueur, d'hémiplégie, de panaris, d'amputation du doigt, de palpitation du cœur, de bouffée de chaleur, d'absence de sensation plantaire, d'Accident Vasculaire Cérébral (AVC) avaient été constatés. Les résultats indiquent aussi que différentes circonstances avaient révélé des complications au cours de bilan de santé à $56,4 \%$, des cas de paludisme à $7 \%$, de fatigue à $4,5 \%$, de vertiges à $2,6 \%$, de furoncle à $2,4 \%$, de gros bébé à l'accouchement à 1,9\%, de bilan opératoire à $1,4 \%$, de maux de tête à $1 \%$ et de rares cas à de toux chronique, de fractures, d'opération de cataracte, de gros ventre, d'angines, d'hémiplégie, de douleurs abdominales, de prostate, de pied enflé, d'ulcère, d'insomnie, d'échauffement de tout le corps, de prurit vaginal, d'assèchement de la bouche à moins de $1 \%$.

\section{Paramètres biologiques de complication du diabète}

Des corrélations significatives ( $p<$ $0,05)$ ont été notées entre l'hypertension artérielle (HTA) et la néphropathie à 0,096 avec $\mathrm{p}=0,021$ ) et à $\mathrm{p}<0,01$ entre 1'HTA et l'hémoglobine glyquée à 0,126 avec $\mathrm{p}=$ 0,002). Des corrélations significatives ont été enregistrées à $\mathrm{p}<0,01$ entre la dyslipidémie et l'artériopathie $(0,332$ avec $\mathrm{p}=0,000)$, la rétinopathie (à 0,109 avec $\mathrm{p}=0,009$ ), la neuropathie (à 0,090 avec $\mathrm{p}=0,030$ ) ; entre l'hyperuricémie et l'artériopathie $(0,147$ avec $\mathrm{p}$ $=0,000)$, à $\mathrm{p}<0,05$ entre l'hyperuricémie et la rétinopathie $(0,088$ avec $\mathrm{p}=0,035)$, entre l'obésité et la néphropathie (à 0,099 avec $\mathrm{p}=$ 0,018 ), la neuropathie (à 0,083 avec $\mathrm{p}=$ 0,046 ), entre l'IMC et la rétinopathie (à 0,103 avec $\mathrm{p}=0,018)$, à $\mathrm{p}<0,01$ entre l'IMC et la 
rétinopathie $(0,108$ avec $\mathrm{p}=0,009)$, à $\mathrm{p}<0,05$ une corrélation significative entre le tour de taille et la néphropathie $(0,085$ avec $\mathrm{p}=$ $0,042)$, la neuropathie $(0,082$ avec $\mathrm{p}=0,049)$; entre la prise d'alcool et la neuropathie $(0,083$ avec $\mathrm{p}=0,046)$, la lésion de pied $(0,090$ avec $\mathrm{p}=0,031$ ), le trouble sexuel (à 0,083 avec $\mathrm{p}=$ 0,047). Egalement une corrélation significative a été signalée à $\mathrm{p}<0,01$ entre l'infection urinaire et l'hémoglobine glyquée (à 0,119 à $\mathrm{p}=0,004$ ), à $\mathrm{p}<0,05$ des corrélations significatives ont été observées entre l'infection urinaire et la glycémie (à
0,088 avec $\mathrm{p}=0,035)$, entre l'antécédent familial et la rétinopathie (à 0,088 avec $\mathrm{p}=$ 0,045), la lésion de pied (à 0,094 avec $\mathrm{p}=$ 0,025), la glycémie (à 0,093 avec $\mathrm{p}=$ 0,026). Les résultats montrent une corrélation significative à $\mathrm{p}<0,01$ entre la position assise prolongée et la néphropathie (à 0,186 avec $\mathrm{p}=$ $0,000)$ aussi des corrélations significatives $\mathrm{p}<$ 0,05 entre la position assise et la rétinopathie (à 0,089 avec $\mathrm{p}=0,033$ ), la neuropathie (à 0,105 avec $\mathrm{p}=0,012$ ), la glycémie (à 0,100 avec $\mathrm{p}=0,017)$. Les résultats sont consignés dans le Tableau 4.

Tableau 1 : La répartition de la population diabétique en fonction des facteurs associés.

\begin{tabular}{|c|c|c|}
\hline $\begin{array}{l}\text { Facteurs associés à } \\
\text { l'apparition du diabète }\end{array}$ & $\begin{array}{c}\text { Variables associées à l'apparition du } \\
\text { diabète }\end{array}$ & $\begin{array}{c}\text { Répartition dans la population } \\
\text { diabétique }(\%)\end{array}$ \\
\hline & $<$ à 35 ans & 5,22 \\
\hline \multirow[t]{2}{*}{ Age } & 35 à 40 ans & 5,57 \\
\hline & $>40$ ans & 89,21 \\
\hline \multirow[t]{4}{*}{ Sexe } & Homme & 33,8 \\
\hline & Femme & 66,2 \\
\hline & Ménagère & 47,2 \\
\hline & Commerçant & 15,6 \\
\hline \multirow[t]{4}{*}{ Profession } & Fonctionnaire & 14,4 \\
\hline & Cultivateur & 13,2 \\
\hline & Retraité & 9,6 \\
\hline & $\leq 100$ & 64,5 \\
\hline \multirow[t]{2}{*}{ Revenu } & $>100$ à $\leq 800$ & 34,4 \\
\hline & $>800$ & 1,0 \\
\hline \multirow[t]{2}{*}{ Antécédent familial } & $I A F$ & 92,5 \\
\hline & Parenté & 7,5 \\
\hline
\end{tabular}

IAF : ignorant antécédent familial, le revenu est estimé en dollars US : 1 \$ = 500 FCFA. 
Tableau 2 : Caractéristiques cliniques et biologiques des patients.

\begin{tabular}{|c|c|c|}
\hline Fonctions biologiques & Valeurs mesurées & Valeurs de référence \\
\hline \multicolumn{3}{|l|}{ Tension artérielle (en $\mathrm{mm} \mathrm{Hg}$ ) } \\
\hline Systole Bras droit & $142,61 \pm 25,22$ & $<130$ \\
\hline Diastole du Bras droit & $82,77 \pm 13,9$ & $<80$ \\
\hline Pouls du bras droit & $83,87 \pm 17,63$ & $60-80$ \\
\hline Systole du Bras gauche & $139,01 \pm 23,95$ & $<130$ \\
\hline Diastole du bras gauche & $80,98 \pm 12,76$ & $<80$ \\
\hline Pouls du bras gauche & $83,59 \pm 17,39$ & $60-80$ \\
\hline \multicolumn{3}{|c|}{ Composantes lipidiques (en mmol/L) } \\
\hline Cholestérol total & $4,92 \pm 1,10$ & $<5,2$ \\
\hline HDL-Cholestérol & $2,35 \pm 0,38$ & $<1$ \\
\hline Triglycérides & $2,45 \pm 0,74$ & $<2,20$ \\
\hline LDL-Cholestérol & $2,08 \pm 0,87$ & $<2$ \\
\hline Acide urique (en $\mu \mathrm{mol} / \mathrm{L})$ - & $352,23 \pm 140,65$ & $180-420$ \\
\hline $\mathrm{HbA} 1 \mathrm{C}($ en $\%)$ & $9,47 \pm 2,41$ & $4-6$ \\
\hline Créatinémie $(\mu \mathrm{mol} / \mathrm{L})$ & $66,82 \pm 20,34$ & $\mathrm{H}(70-120) \mathrm{F}(50-90)$ \\
\hline $\mathrm{IMC}\left(\mathrm{en} \mathrm{kg} / \mathrm{m}^{2}\right)$ & $26,90 \pm 5,80$ & $18,5-24,9$ \\
\hline \multicolumn{3}{|l|}{ Tour de taille $(\mathrm{en} \mathrm{cm})$} \\
\hline Homme & $94.29 \pm 13,02$ & $<94$ \\
\hline Femme & $94.20 \pm 12,65$ & $<80$ \\
\hline Perte de dents & $4,54 \pm 6,41$ & \\
\hline
\end{tabular}

Tableau 3: Corrélation entre les facteurs associés du diabète et les paramètres cliniques et biologiques.

\begin{tabular}{|c|c|c|c|c|c|}
\hline & Age & Sexe & Profession & Revenu & mensuel \\
\hline HTA & $0,340 * *(0,000)$ & - & $0,142 * *(0,001)$ & & - \\
\hline Dyslipidémie & $0,102 * \quad(0,014)$ & - & - & & - \\
\hline $\mathrm{HbA1C}$ & $0,097 * \quad(0,020)$ & - & - & & - \\
\hline Rétinopathie & $0,169 * *(0,000)$ & - & - & & - \\
\hline Créatinémie & $0,107 * \quad(0,010)$ & - & $0,096^{*} \quad(0,021)$ & & - \\
\hline Maux de dent & $0,084 * \quad(0,044)$ & - & - & & - \\
\hline Maux d'oreille & $0,117 * *(0,005)$ & - & - & & - \\
\hline Perte de dents & $0.324 * *(0,000)$ & - & $0,098^{*}(0,019)$ & $0,093 *$ & $(0,027)$ \\
\hline IMC & $0,120 * *(0,004)$ & $0,290 * *(0,000)$ & - & & - \\
\hline Obésité & - & $0,219 * *(0,000)$ & - & & - \\
\hline Tour de taille & - & $0,184 * *(0,000)$ & - & & - \\
\hline Trouble sexuel & - & $0,292 * *(0,000)$ & - & $0,142 * *$ & $*(0,001)$ \\
\hline Protéine de 24 heures & - & $0,093 * \quad(0,026)$ & - & & - \\
\hline Angor / cardiopathie & - & - & - & $0,103 *$ & $(0,013)$ \\
\hline
\end{tabular}


Tableau 4: La corrélation entre les paramètres cliniques-biologiques et les complications du diabète.

\begin{tabular}{|c|c|c|c|c|c|c|c|c|}
\hline & Néphropathie & HbA1C & Artériopathie & Rétinopathie & Neuropathie & Lésion de pied & $\begin{array}{c}\text { Problème lié } \\
\text { au sexe }\end{array}$ & Glycémie \\
\hline HTA & $0,096 *(0,021)$ & $0,126^{* *}(0,002)$ & - & - & - & - & - & - \\
\hline Dyslipidémie & - & - & $0,332 * *(0,000)$ & $0,109 * *(0,009)$ & $0,090 *(0,030)$ & - & - & - \\
\hline Hyperuricémie & - & - & $0,147 * *(0,000)$ & $0,088 *(0,035)$ & - & - & - & - \\
\hline Obésité & $0,099 *(0,018)$ & - & - & - & $0,083 *(0,046)$ & - & - & - \\
\hline IMC & - & $0,103 *(0,013)$ & - & $0,108 * *(0,009)$ & - & - & - & - \\
\hline Tour de taille & $0,085 *(0,042)$ & - & - & - & $0,082 *(0,049)$ & - & - & - \\
\hline Prise d'alcool & - & - & - & - & $0,083^{*}(0,046)$ & $0,090 *(0,031)$ & $0,083 *(0,047)$ & - \\
\hline Prise de tabac & - & $0,100 *(0,017)$ & - & - & - & - & - & - \\
\hline Infection urinaire & & $0,119 * *(0,004)$ & - & - & - & - & - & $0,088 *(0,035)$ \\
\hline Antécédent familial & - & - & - & $0,084 *(0,045)$ & - & $0,094 *(0,025)$ & - & $0,093 *(0,026)$ \\
\hline Position assise & $0,186^{* *}(0,000)$ & - & & $0,089 *(0,033)$ & $0,105 *(0,012)$ & - & - & $0,100 *(0,017)$ \\
\hline
\end{tabular}

** veut dire que la corrélation est positive et significative au seuil $\mathrm{p} \leq 0,01$

* veut dire que la corrélation est positive et significative au niveau de $\mathrm{p} \leq 0,05$. 


\section{DISCUSSION}

\section{Causes de l'apparition du diabète}

Notre étude a révélé que l'âge, le sexe et la profession sont des facteurs directement liés à la manifestation de la maladie au Burkina Faso. La tranche de patients de plus 40 ans d'âge est extrêmement touchée, cela s'explique par le vieillissement qui s'accompagne de modification du métabolisme glucidique, favorisant la survenue du diabète : diminution de l'insulinosécrétion. En effet, les cellules $\beta$ du pancréas répondent moins bien aux stimuli glucidiques et par la diminution de la sécrétion des hormones contre la régularisation, notamment du glucagon (Fattorusso et Ritter, 2004). La diminution de la sensibilité à l'insuline s'explique en partie par des modifications de la composition corporelle avec diminution de la masse maigre et augmentation de la masse grasse (Haslett, 2004). Selon notre étude, 66\% des femmes en âge avancé sont obèses ou ayant un surplus de poids $26,90 \pm 5,80 \mathrm{~kg} / \mathrm{m}^{2}$, par conséquent, renferment généralement assez de masse grasse. Dans notre étude, beaucoup de femmes ont été victimes de cette maladie et parfaitement ignoraient la survenance ou l'apparition du diabète contrairement aux mères impliquées dans l'étude d'Angleterre (Whitehead et Dimmock, 2013).

Notre étude a montré que parmi les diabétiques de plus de 40 ans, 89\% avaient des ascendants familiaux diabétiques, d'où l'intérêt de mener un dépistage et une éducation dans les familles. L'ignorance de l'antécédent familial et de surcroît, l'incapacité d'effectuer des analyses biologiques assez régulièrement, dû au coût élevé des examens biologiques et à la faiblesse du revenu des ménages, expliquent nos résultats. Une étude réalisée en Angleterre (Whitehead et Dimmock, 2013) indique qu'il faut un dépistage précoce, ce qui n'est pas le cas au Burkina Faso. Pour éviter des complications futures de la maladie qui est silencieuse, un dépistage précoce est à effectuer lors des inscriptions dans les structures de formations d'éducations et lors des recrutements pour un poste de travail, ce qui permet de les prendre en charge dès lors qu'ils sont positifs au diabète sucré.

Notre étude révèle que la profession exercée par les uns et les autres (ménagères, commerçants, agents de bureau et retraités) conduit à la sédentarité; la tranche d'âge $<40$ ans est autour de $11 \%$; la majorité des patients était pauvre et $64,5 \%$ ont eu un revenu < à $100 \$$. Ces résultats indiquent qu'aujourd'hui le diabète de type 2 n'apparaît pas seulement chez les personnes aisées mais aussi chez les personnes à revenu modeste car un problème de malnutrition s'impose (Townsend et al., 2001). La maladie se retrouve aussi bien chez les jeunes, groupe à haut risque en raison du changement brusque de leur mode de vie (Kacenelenbogen, 2006 ; Hillier et Pedula, 2003). Cette sédentarité et malnutrition conduisent à un surpoids et particulièrement à l'accumulation de gras dans les organes de l'abdomen qui entraînent une résistance à l'insuline (Grimaldi et al., 1998). Dans notre étude, nous avons eu le tour de taille de 94, $29 \pm 13,02 \mathrm{~cm}$ chez l'homme et de $94,29 \pm 12,65 \mathrm{~cm}$ chez la femme, ce qui traduit l'état d'accumulation de gras au niveau du ventre. Une étude au Bénin a constaté que l'obésité abdominale a été associée à la plus grande probabilité du syndrome métabolique (Ntandou et al., 2009). L'obésité abdominale s'est également avérée être un facteur de risque important pour l'arrêt du cœur parmi des adultes au Congo, où les adultes avec des rapports accrus de taille-à-hanche avaient augmenté le risque de l'arrêt du cœur (Rabasa-Lhoret et Laville, 2003). Selon l'IDF (2009), 80 à 90\% des personnes atteintes de diabète de type 2 ont un excès de poids ou sont obèses. Ces deux pathologies sont responsables d'une augmentation significative de la mortalité (Longo-Mbenza et al., 2007). Il convient alors de réaliser une enquête alimentaire pragmatique pour dépister des anomalies qualitatives et quantitatives de l'alimentation auprès de chaque patient suite à un bilan biologique. 


\section{Causes de la complication du diabète}

Les résultats ont montré une corrélation positive entre l'artériopathie et la dyslipidémie et entre l'hyperuricémie. L'hyperuricémie provient de la dégradation métabolique exagérée de la purine au niveau du foie et excrétée par les reins (Toshihisa et al., 2013). L'élévation de cet acide urique résulte du développement de la goutte (artériopathie) et pourrait être un facteur de risque pour les maladies cardiovasculaires et le diabète. Il est à noter que la dyslipidémie serait responsable de l'artériosclérose des maladies vasculaires. En plus de ces observations, nous remarquons dans notre étude qu'aussi, l'hémoglobine glyquée (déséquilibrée à 9,47 $\pm 2,41 \%$ ), l'artériopathie, la néphropathie, les troubles sexuels, les lésions du pied et la glycémie ont une corrélation quelques fois avec les différents facteurs de risques tels que : hyperuricémie, Indice de Masse Corporelle (IMC), tour de taille, la prise d'alcool, prise de tabac, les infections urinaires, antécédent familial et la position assise. Une glycémie élevée de façon chronique endommage peu à peu les nerfs et les vaisseaux sanguins, principalement les yeux et les reins. Le diabète peut ainsi être à l'origine de maladies cardiovasculaires, d'une perte de vision irréversible, de douleurs en raison d'atteintes des nerfs ou d'insuffisance rénale, de troubles sexuels (dysfonctionnement érectile chez l'homme et sécheresse vaginale chez la femme). Ce phénomène explique pourquoi généralement, les complications du diabète de type 2 ne surviennent qu'après plusieurs années de vie. Ces complications sont très souvent attribuées à d'autres maladies telles que le paludisme, les maux de tête, les vertiges, la furonculose que nous avions rencontré au cours de notre étude. La découverte de ce diabète dans notre étude est fortuite à $100 \%$ car elle a été décelée au cours d'une visite médicale ou d'un bilan d'examens biologiques. Nos résultats corroborent ceux obtenus par Song et Hardisty (2009) où les complications du diabète reposaient sur la neuropathie, la rétinopathie. Ces complications ont une corrélation avec l'hypertension artérielle (HTA), la dyslipidémie, le poids, le contrôle de la glycémie et l'obésité. Il est à noter que l'hypertension artérielle contribuerait probablement à l'aggravation et à la précocité des complications.

D'après les résultats obtenus par Song et Hardisty (2009) en Angleterre, les complications microvasculaires débutent de 13 à 20 ans avant son déclenchement. Le tabac n'agit pas directement sur le diabète de type 2 mais, il intervient pour accroître le risque de crise cardiaque ou d'accident vasculaire cérébral, car il endommage les vaisseaux sanguins (Hatunic et al., 2005) pour augmenter le risque d'atteintes nerveuses et de problèmes rénaux et par conséquent accroître le taux de glycémie. En fait, la nicotine freine l'efficacité de l'insuline, un syndrome appelé « résistance à l'insuline » (Grimaldi et al., 1998). Il peut compliquer la gestion du diabète et entraîner une prédisposition aux rhumes, aux infections et des problèmes d'érection (Said, 1999). Par ailleurs, il n'y avait pas eu de corrélation entre le $\mathrm{HbA1C}$ et l'hyperuricémie comme avait trouvé en Iran Rafieian-Kopaei et al. (2014). Par contre, nous avons eu dans notre étude une corrélation entre l'HTA et l'HbAlC $(r=0.126 ; p=$ $0,002)$. Ce qui signifierait que pour éviter les complications du diabète, il faut forcément que la Tension artérielle soit normale c'est-àdire < à $130 / 80$ mm Hg.

\section{Méthode de traitement conventionnel et non conventionnel (nouvelle stratégie du traitement du diabète)}

De notre étude, $92,5 \%$ des enquêtés ne savent pas l'existence ou non de cette maladie auprès de leur parent. Il est conseillé de connaître les antécédents médicaux des parents afin de prévenir la maladie. Il est préférable de diagnostiquer le diabète à temps du lieu d'être déçu à la fin avec les complications.

Les manifestations de goutte, de picotement, de crampes et d'échauffement 
plantaire obtenues au cours de notre étude (consignés dans le Tableau 2) proviendraient, notamment des problèmes de lipide (dyslipidémie) et de l'hyperuricémie. Les mêmes phénomènes ont été constatés par Hadjeres et al. (2009). La maîtrise de la consommation des aliments riches en matières grasses et en purine sauf le poisson, limiterait ou retarderait l'apparition de l'artériopathie qui constitue un facteur de risque dans la survenue de la neuropathie à travers les manifestations de goutte, de picotement et échauffement plantaire, les crampes (Said, 1999). La surveillance de cette artériopathie retarderait ou éviterait les complications au niveau des pieds qui devraient aboutir au pire des cas aux pieds diabétiques voire une amputation en cas de gangrène (Sano et al., 1999 ; Ouedraogo et al., 2000). Nous pouvons dire qu'il est conseillé de proscrire des habitudes alimentaires malsaines, beaucoup de crudité dans le but de prévenir les complications de santé supplémentaires. Afin d'être sûr que le diabète est sous contrôle, il est conseillé d'avoir des contrôles réguliers au niveau dentaire, oculaire et le taux de sucre dans le sang, et connaître les antécédents médicaux des parents. Signaler au médecin pour chaque affection bénigne qui peut être rencontrée, car il peut être causé par la maladie.

Le surpoids et l'obésité constatés chez les patients dans notre étude prouvent que ces patients n'utilisent pas l'activité physique comme un traitement adéquat de la maladie. Il est conseillé d'avoir un quotidien de 60 minutes d'exercice pendant 5 jours par semaine pour permettre à l'organisme d'utiliser le sucre du sang sous forme d'énergie et aussi pour se débarrasser de la masse graisseuse qui serait à l'origine de la résistance de l'insuline. C'est une pratique très importante, car l'inactivité du corps est considérée comme l'une des raisons pour le diabète de type 2 . L'exercice doit inclure la marche rapide, le jogging, le vélo, la natation ou de tout autre travail qui sera fonction des préférences des patients.

\section{Conclusion}

On peut conclure que l'âge, le sexe et la profession sont des facteurs directement liés à la manifestation de la maladie à BoboDioulasso.

Parmi les causes de complications du diabète, la dyslipidémie et l'hyperuricémie constituent les causes principales de l'artériopathie par laquelle surviennent l'hypertension artérielle, la néphropathie et la neuropathie qui par conséquent provoque la rétinopathie. Toute affection bénigne doit être signalée le plus rapidement possible aux praticiens.

La connaissance des antécédents médicaux des parents, une alimentation saine et équilibrée, la surveillance du poids par la pratique régulière d'une activité, physique permettront de prévenir, de retarder ou d'éviter les complications du diabète du type 2.

\section{REMERCIEMENTS}

Nos remerciements au comité d'éthique pour sa disponibilité et son apport, également à tous les personnels du LARESBA, du CHUSS de Bobo-Dioulasso, du dispensaire Burkina secours et du Centre Médical Social Don Bosco pour leur apport très louable et sans oublier les patients diabétiques pour leur contribution à cette étude.

\section{REFERENCES}

Drabo J, Sidibé A, Halimi S, Besançon S. 2011. Une approche multipartenaire du développement de l'excellence dans la formation à la gestion du diabète dans quatre pays africains. Diabetes Voice, 56(1): 4-48.

Fattorusso V, Ritter O. 2004. Maladies du métabolisme et de la nutrition. In Vadémécum Clinique : du Diagnostic au Traitement (17é édn). Fattorusso V, Ritter O. MASSON : Paris ; 802-821.

Grimaldi A, Cornet P, Masseboeuf N, Popelier M, Sachon C. 1998. Guide Pratique du Diabète. Editons Médicales Spécialisées : José Vieira ; 376p. 
Haslett-E C. 2004. Médecine Interne Principes et Pratiques (Traduit de la $19^{\text {ème }}$ Edition Anglaise). Maloine : Paris, 1200p.

Hatunic M, Burns N , Finucane F, Mannion C , Nolan JJ . 2005. Contrastées état de risque clinique et cardio-vasculaires entre le début et le type 2 diabète plus tard, l'apparition. Diabète Vasc. Dis. Res., 2: $73-75$.

Hillier TA, Pedula KL. 2003. Complications chez les jeunes adultes de type 2 diabète à début précoce: la perte de la protection relative de la jeunesse. Diabetes Care, 26: $2999-3005$.

Horde P. www.sante-medecine.net. 2011. Dernière modification le lundi 31 janvier 2011 à 12:54:07.

IDF (International Diabetes Federation). 2009. Number of people with diabetes (20-79 years) 2010 - 2030. Diabetes Atlas ( $4^{\text {th }}$ edn), IDF.

Kacenelenbogen N. 2006. Weight management in general practice. Rev. Med. Brux., 27: S 361 - 371

Longo-Mbenza B, Mambune HF, Kasiamm JB, Vita EK, Fuele SM, Nsenga JN, Mabwa L, Nzuzi V. 2007. Relationship between waist circumference and cholesterol in Central Africans with congestive heart failure. West Afr. J. Med., 26(3): 183-190.

Ntandou G, Delisle H, Agueh V, Fayomi B. 2009. Abdominal obesity explains the positive rural-urban gradient in the prevalence of the metabolic syndrome in Benin, West Africa. Nutr Res., 29(3): 180-189.

Ouedraogo M, Ouedraogo SM, Birba E, Drabo YJ. 2000. Complications aiguës du diabète sucré au Centre Hospitalier Nationale Yalgado Ouédraogo. Médecine d'Afrique Noire, 47(12): 505-507.

Rabasa-Lhoret R, Laville M. 2003. Physiopathologie des obésités et du diabète de type 2. Encycl Méd Chir, Endocrinologie-Nutrition. Elsevier : Paris.

Rafieian-Kopaei M, Behradmanesh S, Kheiri S, Nasri H1. 2014. Association of serum uric Acid with level of blood pressure in type 2 diabetic patients. Iran J. Kidney Dis. Mar., 8(2):152-154.

Said G. 1999. Neuropathies Diabétiques. Encycl Méd Chir, Elsevier : Paris.

Sano D, Thieno H, Drabo Y, Sanou A. 1999. Prise en charge du pied diabétique à propos de 42 cas au CHU de Ouagadougou. Médecine d'Afrique Noire, 46(6): 157-174.

Song SH, Hardisty CA. 2009. Early onset type 2 diabetes mellitus: a harbinger for complications in later years- Clinical observation from a secondary care cohort. Q. J. Med., 102: 799 - 806.

Toshihisa I, Wanping AW, Kiyoko K. 2013. Metabolic Interactions of Purine Derivatives with Human ABC Transporter ABCG2: Genetic Testing to Assess Gout Risk. Pharmaceuticals, 6: 1347-1360.

Townsend M, Peerson J, Love B, Achterberg C, Murphy S. 2001. Food insecurity is positively related to overweight in women. J. Nutr., 131: 1738-1745.

Whitehead AL, Dimmock M, Place M. 2013. Diabetes control and the influence of family functioning. Journal of Diabetes Research and Clinical Metabolism, 2(16): 157-174. 